\title{
Superconducting intermediate state of white tin studied by muon-spin-rotation spectroscopy
}

\author{
V. S. Egorov, ${ }^{1}$ G. Solt,${ }^{2}$ C. Baines, ${ }^{2}$ D. Herlach, ${ }^{2}$ and U. Zimmermann ${ }^{2}$ \\ ${ }^{1}$ Russian Research Center “Kurchatov Institute,"' Moscow 123182, Russia \\ ${ }^{2}$ Paul Scherrer Institut, CH-5232 Villigen PSI, Switzerland
}

(Received 9 October 2000; revised manuscript received 27 December 2000; published 21 June 2001)

\begin{abstract}
The local magnetic field in the bulk of superconducting single crystal $\beta$ tin was measured near and across the transition between the normal $(N)$ and intermediate $(I)$ state at $T=0.08 \mathrm{~K}$. With varying applied field $H_{a}$, the data indicate a reversible phase transition within the $I$ state at $H_{t} \approx 295 \mathrm{Oe}$, interpreted as a transition from the laminar to a tubular structure. Near the $I \rightarrow N$ transition, occurring at $H_{c I}=0.98 H_{c 0}=300$ Oe, the field $B$ in the $N$ domains is strongly inhomogeneous with $\bar{B}<H_{c 0}$, it grows with the decrease of $H_{a}$ towards the value $H_{c 0}=305.5 \pm 0.5$ Oe. On lowering $H_{a}$ in the $N$ state, a large "field supercooling" $\left(\delta H / H_{c} \approx 6 \%\right)$ of this state was observed.
\end{abstract}

DOI: 10.1103/PhysRevB.64.024524

PACS number(s): 74.55. $+\mathrm{h}, 74.25 . \mathrm{Ha}, 76.75 .+\mathrm{i}$

\section{INTRODUCTION}

Superconducting $(S)$ and normal $(N)$ domains in type 1 superconductors coexist in the intermediate $(I)$ state, in a first approximation, for applied fields $\mathbf{H}_{a}$ within the range (1 $-n) H_{c}<H_{a}<H_{c}(n=$ demagnetization factor). Taking into account the energy of interfaces between $N$ and $S$ domains, ${ }^{1}$ the $N-I$ transition should occur at a field $H_{c I}<H_{c}$ (Ref. 2) for which, in the case of a plate oriented perpendicular to the field and assuming a laminar domain structure, the calculation $^{3,4}$ gives

$$
H_{c I} \approx H_{c}\left[1-2 \theta(\delta / d)^{1 / 2}\right], \quad \theta=\sqrt{\ln 2 / \pi} \approx 0.47,
$$

where $d$ is the sample thickness and $\delta \approx \xi-\lambda_{L}$ is determined by the correlation and penetration lengths $\xi$ and $\lambda_{L}$. The same calculation predicts ${ }^{3}$ that the average field $\bar{B}$ in the $N$ domains is somewhat lower than $H_{c}$, having its lowest value $B=H_{c I}$ at the $N-I$ transition, and growing towards $H_{c}$ with the decrease of $H_{a}$. Earlier experiments ${ }^{5,6}$ have qualitatively confirmed Eq. (1), but no information is available on the predicted depression of $B$ in the $N$ domains. This is partly due to imperfections in the samples, causing "tails" in the magnetization curve. ${ }^{6}$ Also, in the earlier experiments no direct, spectroscopic data from within the bulk material could be obtained.

As to the spatial structure of the $I$ state, theoretical treatments normally deal with straight, regular $S$ and $N$ laminae parallel to $\mathbf{H}_{a} \cdot{ }^{1,3,4,7}$ Though these are seen in "pure form", only in special cases, even the most often observed intricate patterns, ${ }^{5,8-10}$ rich in topological variations, are indeed various "corrugated" forms of a laminar structure. However, near the $N-I$ (or $I-S$ ) transition, where the $S$ (or $N$ ) phase nucleates and extends over only a very small fraction of the volume, a threadlike structure for the minority phase may be more advantageous. ${ }^{11,2}$ Experimentally, a system of superconducting tubes emerging on the sample surface were observed, e.g., in $\mathrm{Pb}, \mathrm{Sn}$ (Ref. 12), and $\mathrm{Al}$ (Ref. 5) for $H_{a}$ $\lesssim H_{c}$, near the $I \rightarrow N$ transition.
For measuring the local magnetic field deep in the bulk of the $N$ domains, the unique practicable method is muon spin rotation $(\mu \mathrm{SR})$ spectroscopy. In the few previous $\mu \mathrm{SR}$ studies of type 1 superconductors, ${ }^{13,14}$ however, no results on the $I-N$ transition in single crystal samples have been reported. [In Ref. 14 the $N-I$ transition in polycrystalline lead was studied at an elongated sample directed along the field, not suited to test Eq. (1).]

The results of $\mu \mathrm{SR}$ measurements near and through the $N-I$ transition in a plate of single crystal white $(\beta-)$ tin are presented below. (Preliminary data, obtained with a field mesh too coarse for a detailed study of the transition region, were reported in Ref. 15.)

\section{EXPERIMENT}

The ultrahigh purity $\mathrm{Sn}$ single crystal $[18 \times 12$ $\times 0.56 \mathrm{~mm}^{3}$, electron mean free path $\approx$ some $\mathrm{mm}$ (Ref. 16)] was a plate, grown parallel to the (100) plane. The sample was oriented perpendicular to the homogeneous magnetic field $\mathbf{H}_{a}$ and held at the temperature $T=0.08 \mathrm{~K}$, the direction of the incident $\mu^{+}$beam was parallel to the field. A plate of polycrystalline tin with dimensions similar to the single crystal was also used for comparison.

The $\mu^{+}$particle has spin $S=1 / 2$ and a magnetic moment $\mu_{\mu}=3.183 \mu_{p}$, it decays with a lifetime of $\tau_{\mu}=2.20 \mu$ s into a positron and two neutrinos. In the transverse-field $\mu \mathrm{SR}$ experiment ${ }^{17}$ the precessing polarization $\mathbf{P}(t)$ of the muons stopped within the sample is monitored. The polarization $\mathbf{P}(0)$ at the moment of the implantation $(t=0)$ is known, and its instantaneous direction and magnitude at later times are revealed by the parity-breaking, anisotropic momentum distribution of the decay positrons escaping from the sample. The $\mu^{+}$spin in $N$ domains precesses with the frequency $\nu$ $=\left(\gamma_{\mu} / 2 \pi\right) B$ determined by the local field $B \quad\left(\gamma_{\mu}=2 \pi\right.$ $\times 13.554 \mathrm{kHz} \cdot \mathrm{G}^{-1}$ is the gyromagnetic ratio of $\left.\mu^{+}\right)$, the frequency for muons in $S$ domains is zero. Stopping and subsequent decay of a large number of muons are detected, and the number of decay positrons $N_{x}(t)$ emerging in a given 


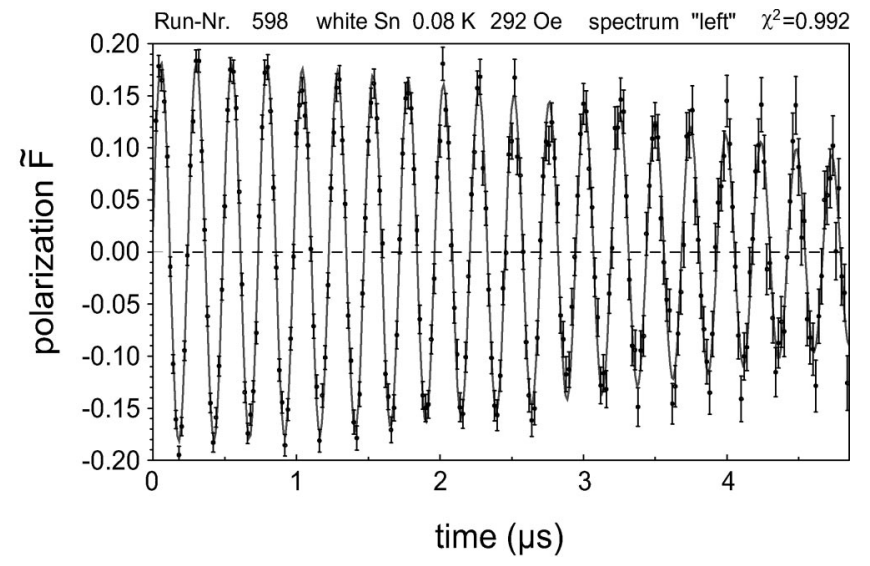

FIG. 1. Histogram of the polarization $\widetilde{F}_{l}=a_{N} \widetilde{P}_{x}(t)$ measured at $T=0.08 \mathrm{~K}, H_{a}=292$ Oe by detector $l$. The fit (solid line) gives $\nu=\left(\gamma_{\mu} / 2 \pi\right) B=4.0653 \pm 0.0008 \mathrm{MHz}, a_{N}=0.182 \pm 0.002$, $\sigma=0.246 \pm 0.004 \mu \mathrm{s}^{-1}$.

direction (taken as the $x$ axis) is proportional to $P_{x}(t)$, showing an oscillating component $\widetilde{P}_{x}$ with frequency $\bar{\nu}$. The damping rate (Gaussian, exponential, or other) of $\widetilde{P}$ characterizes the spread of frequencies about $\bar{\nu}$, and the oscillation amplitude $a_{N}$ at $t=0$ (called "asymmetry") is proportional to the number of muons stopped in $N$ domains. Therefore, since the muons are uniformly implanted over $N$ and $S$ regions, one has $a_{N} \propto \rho_{N}\left(H_{a}\right)$ (the amplitude $a_{S} \propto \rho_{S}$ for the muons in $S$ domains appears as the "missing asymmetry" in these measurements).

The experiments were performed at the low temperature facility LTF on the $\pi \mathrm{M} 3$ beamline of PSI, Villigen, with the accidental background reduced by use of the MORE (muonson-request) technique. ${ }^{18}$ The penetration range of the $\mu^{+}$ beam in the $\mathrm{Sn}$ sample was $\approx 0.1 \mathrm{~mm}$, ensuring that practically all muons stop in the bulk of the specimen.

\section{RESULTS}

After division by the trivial lifetime factor $e^{-t / \tau_{\mu}}$, the oscillating $\mu$ SR signal is proportional to $a_{N} \widetilde{P}_{x}(t) \equiv \widetilde{F}(t)$. To describe the damping of $\widetilde{P}_{x}$, the Gaussian form was overall found to be the most adequate, even if a more complex behavior is also visible near the transition region. For a Gaussian decay of the polarization one has

$$
\widetilde{F}_{i}\left(t ; H_{a}\right)=a_{N}\left(H_{a}\right) e^{-(1 / 2)\left[\sigma\left(H_{a}\right) t\right]^{2}} \cos \left[\gamma_{\mu} B\left(H_{a}\right) t+\psi_{i}\right],
$$

where the local field $B$, the damping rate $\sigma$ and the "effective asymmetry" $a_{N}$ are to be found by data fitting. The phase $\psi_{i}$ depends on the position of detector $i$, whereas $a_{N}$ was approximately the same for the two detectors "left" and "right" $(i=l, r)$.

Figure 1 shows a typical histogram $\widetilde{F}(t)$ representing $\approx 3 \times 10^{6} \mu^{+}$decay events, together with the fit by Eq. (2), at $H_{a}=292$ Oe. The crystal was in the $I$ state not far from

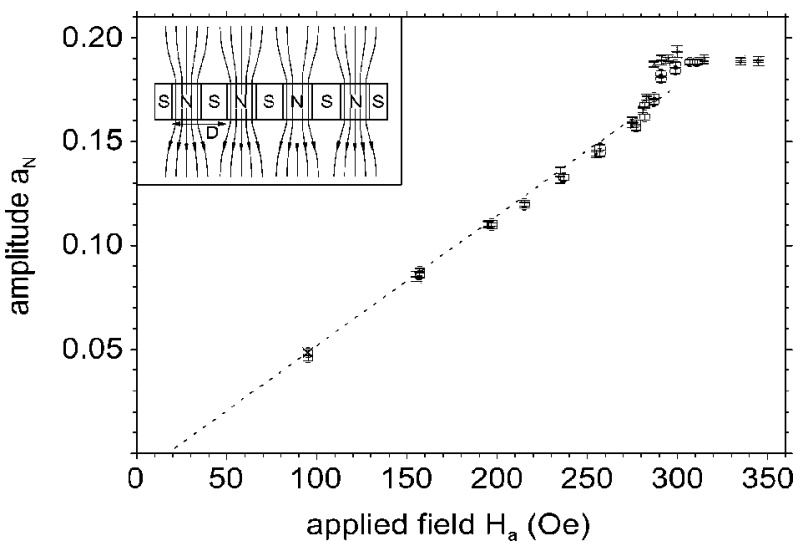

FIG. 2. Amplitude (asymmetry) $a_{N} \propto \rho_{N}\left(H_{a}\right)$ for muons in the $N$ domains, the symbols $\bigcirc$ and $\times$ stand for upward and downward runs. The straight line is a guide to the eye, the extrapolation for the $I \rightarrow S$ transition gives $H_{a S}=18 \pm 3$ Oe. In the field-downwards run, $a_{N} \approx a_{0}$ is still valid below $H_{c I}=300$ Oe down to $\approx 287$ Oe, where the $N$ state persists field supercooled (see Fig. 3). The field distribution in the laminar $I$ state is shown schematically as an inset.

the transition point, the frequency $\nu=\left(\gamma_{\mu} / 2 \pi\right) B$ $=4.0653 \mathrm{MHz}$ corresponds to $\bar{B}=299 \mathrm{G}$.

Since $a_{N} \propto \rho_{N}$, one expects $a_{N}$ to be a constant $a_{0}$ for the uniform $N$ state and decrease linearly with decreasing $H_{a}$ in the $I$ state (the difference $a_{S}=a_{0}-a_{N}$, the missing asymmetry, shows the growing volume of $S$ domains.) This predicted behavior of $a_{N}\left(H_{a}\right)$ is seen in Fig. 2, showing $a_{N}=a_{0}$ $\approx 0.19$ for $H_{a}>300$ Oe and a fairly linear decrease of $a_{N}$ for $H_{a}<290$ Oe. (The nonlinear behavior even for upward runs right below the $N-I$ transition is an artifact of the assumption of simple Gaussian damping also at this field range, discussed in connection with Fig. 4 below.) The extrapolation gives $H_{a}=H_{a S} \approx 18 \pm 3$ Oe for the $I \rightarrow S$ transition where $a_{N}=0$, a fair agreement with the theoretical value $H_{a S}=(1-n) H_{c}$ in view of $n \approx 0.95$ as demagnetizing factor for the present sample geometry. ${ }^{19}$ No data were taken for $H_{a}<95 \mathrm{Oe}$, since with $\rho_{N}, a_{N} \rightarrow 0$ the time required for collecting the decay events needed for a reasonable statistical accuracy becomes extremely long.

The variation of $B$ in the $N$ domains near the $N-I$ transition is shown in Fig. 3 both for decreasing and increasing $H_{a}$. As $H_{a}$ decreases, the uniform $N$ state is metastable and persists supercooled until $H_{a}=H_{c I^{\prime}} \approx 287 \mathrm{Oe}$, which is to be compared to $H_{c 0}\left[1-\left(T / T_{c}\right)^{2}\right] \approx H_{c 0}$ at the sample temperature $T=0.08 \mathrm{~K}$ [the tabulated values are $H_{c 0}=305 \pm 2$ Oe (Ref. 20) and 308 Oe, (Ref. 21) $\left.T_{c}=3.722 \mathrm{~K}\right]$. The discontinuous jump at $H_{c I^{\prime}}$ signals the formation of the $I$ state, but $\bar{B}$ remains well below $H_{c}$ and increases slowly with decreasing $H_{a}$, reaching a plateau only at $H_{a} \lesssim 260$ Oe. The position $B \approx 305.5 \pm 0.5 \mathrm{G}$ of this plateau (observed down to $H_{a}=100$ Oe not shown in the figure) is the " $\mu$ SR value" for $H_{c 0}$, measured within the bulk of $N$ domains.

Crossing the transition from the $I$ state, the monotonic decrease of $\bar{B}$ with increasing $H_{a}$ continues up to $H_{a}=H_{t}$ $\approx 295$ Oe. At this point the curve $\bar{B}\left(H_{a}\right)$ has a cusp, which we interpret as an indication for the phase transition within 


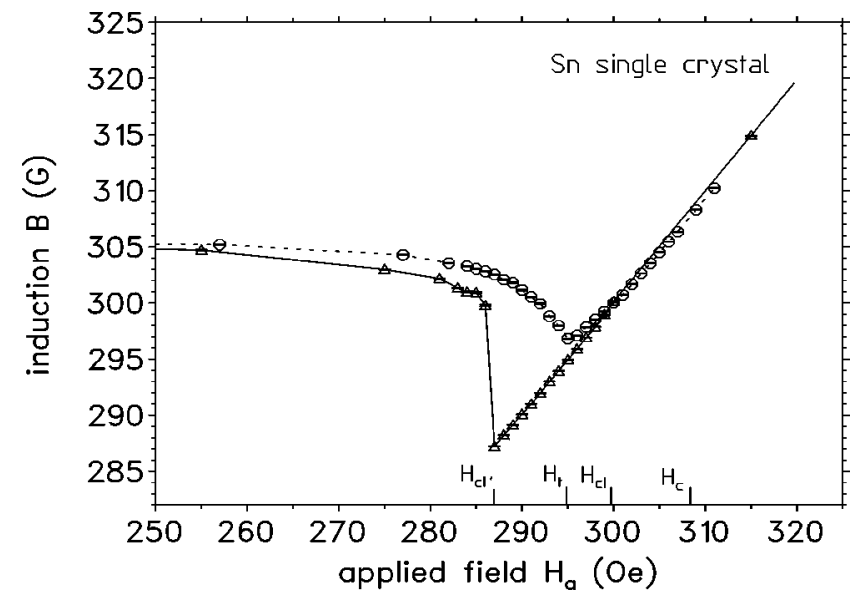

FIG. 3. Field $\bar{B} v s$ applied field $H_{a}$ in the $I-N$ transition region for the Sn single crystal plate at $T=0.08 \mathrm{~K}, \mathbf{H}_{a} \|[100]$. For decreasing $H_{a}$ (solid line) the $N$ state is field supercooled down to $H_{c I^{\prime}} \approx 287$ Oe. On increasing $H_{a}$ (dashed line) the laminar $I$ structure transforms to one with tubular $S$ regions at $H_{t} \approx 295 \mathrm{Oe}$, and the $I \rightarrow N$ transition occurs at a higher field $H_{c I} \approx 300$ Oe. The position of the plateau at $B=305.5 \pm 0.5 \mathrm{G}$ gives the " $\mu$ SR spectroscopic" value for $H_{c}$.

the $S$ phase: as the volume fraction $\rho_{S}$ of the superconducting phase becomes very small, the very thin $S$ laminae become unstable and transform into a structure with threads of superconducting tubes ("antivortices") parallel to $\mathbf{H}_{a}$. On further increasing the field, the tubular $I$ state is seen to go over to the $N$ state smoothly at $H_{a}=H_{c I}=300 \pm 1$ Oe. As long as $H_{a}<H_{c I}$, the upper path in Fig. 3 is completely reversible. [On starting from the uniform $N$ state and lowering $H_{a}$ the tubular structure does not arise, since it is stable only for $H_{t} \leqslant H_{a} \leqslant H_{c I}$, a field range within the interval $\left(H_{c I^{\prime}}, H_{c}\right)$ where the field-supercooled $N$ state persists.]

Although the tubular-laminar transformation was considered already by Landau ${ }^{11}$ and later observed ${ }^{5,12}$ in a few cases, previous sophisticated model calculations made also clear that the free energy differences between different $a$ priori possible spatial structures of the $I$ state are very small, and the actual appearence of one or the other structure is "depending on the exact experimental conditions and the sample quality." 3 To give quantitative predictions concerning $H_{t}$ or $H_{c I}$ for a given sample is therefore prohibitively difficult and will not be attempted here. The simple model below, predicting a tubular-laminar transition, is intended to make plausible how the sequence of two phase transitions $I$ (laminar) $\rightarrow I$ (tubular) $\rightarrow N$ state can arise, depending on the parameters $H_{c}, \delta$, and sample thickness $d$.

On approaching the $I \rightarrow N$ transition, $\rho_{S} \rightarrow 0$ and the period $D$ of the laminar structure increases ${ }^{3}$ as $D$ $\approx(d \delta)^{1 / 2} / \rho_{S} \rho_{N}$. One can thus consider the energy balance for a single $S$ slab of width $2 R$ inserted into the $N$ phase, with the result that it is stable so long as $R / \delta \geqslant 1 /[2(1-h)]$, where $h=H_{a} / H_{c}$ and $1-h \ll 1$. However, $R$ has also an upper limit, since the $S$ slab at the given sample thickness $d$ has ${ }^{19}$ a demagnetizing factor $n \approx 2 R / d$ and it can remain superconducting only so long ${ }^{3}$ as $n<1-h$. (It is assumed that $R \ll d$, and the estimate is for an ellipsoidal shape.) These two conditions can be satisfied if $1-h \geqslant \sqrt{\delta / d}$, in good agreement with Eq. (1) (only with a factor 1 instead of 0.94), and the minimum width for the lamina is $2 R=\sqrt{d \delta}$.

The same argument for a superconducting cylinder of radius $R$ leads to the conditions $\delta / R \leqslant 1-h$ and $n<1-h$ similar to those for the lamina, only technically more complicated because of the expression $n=[\ln (2 q)-1] / q^{2}$ with $q$ $=d / 2 R \gg 1$ for the inscribed ellipsoid in this case. ${ }^{19}$ The two inequalities determine the minimal radius as $R=d / f(\delta / d)$, where $f$ is given by the implicit equation

$$
f^{3}=4 d \ln (f-1) / \delta
$$

and the maximal value of $h$ where the tubular "antivortex" appears is $1-h=\delta f / d$. With the values $\delta \approx 10^{-4} \mathrm{~cm}$ (Ref. 3) and $d=0.056 \mathrm{~cm}$, one obtains $f \approx 18.6$ and the minimal values of $1-h$ where the two structures become stable, are

$$
1-h=0.033 \text { (tube) or } 0.042 \text { (lamina) }
$$

The highest field allowing the nucleation of an $S$ tube is therefore $H_{a}=H_{c I}=0.967 H_{c}$, higher than that for a lamina, which is stable only below $H_{l}=0.958 H_{c}$. This means that the $I \rightarrow N$ transition takes place at $H_{c I}$, from an $I$ state with superconducting tubes. However, for $H_{a}$ not too close to $H_{c}$ the laminar phase is known to have the lower free energy, ${ }^{3}$ therefore a topological transition between tubular and laminar phases is predicted at some field $H_{t}$, which should be near to the "nucleation" value $H_{l}$ calculated above.

Repeated field runs showed that both the field-upwards and field-downwards (supercooling) routes are reproducible. The predicted depression of $H_{c}$ for $\delta \approx 10^{-4} \mathrm{~cm}$ and $d$ $=0.56 \mathrm{~mm}[4 \%$ by Eq. (1) and $3.3 \%$ by Eq. (3)] is in reasonable agreement with $H_{c I} / H_{c} \approx 0.022$ inferred from Fig. 3 . The observed value $\left(H_{c I}-H_{t}\right) / H_{c} \approx 0.016$ is to be compared with the estimate $0.042-0.033=0.009$ through Eq. (3), found by comparing the fields where a single tube or lamina becomes stable in a pure $N$ environment.

The development of the $I$ state with the decrease of the $S$ fraction leads to a large, asymmetric peak of the damping rate $\sigma$ near $H_{a} \approx H_{t}$, about four times its "baseline" value $\sigma_{0}$ measured in the $N$ state, as shown in Fig. 4. (The small but finite $\sigma_{0} \approx 0.06 \mu \mathrm{s}^{-1}$ is due, in addition to instrumental inhomogeneities of $H_{a}$, to fields of the disordered moments of Sn nuclei having nonzero spin and present with a natural abundance of $16.5 \%$. This value of $\sigma_{0}$ corresponds to a dipolar field spread of $\leqslant 0.7$ G.)

Consider first the field range above $\approx 260$ Oe. On increasing $H_{a}$ from this value, $\sigma$ begins to increase from $\sigma_{I}$ $\approx 0.09 \mu \mathrm{s}^{-1}$ and reaches a peak at somewhat below $H_{a}$ $=H_{t}$, then drops abruptly, with the largest slope at $H_{t}$, down to $\sigma_{0}$ at $H_{c I}$. The increase of $\sigma$ with $H_{a}$ on the left side of the peak reflects the increasing effective width of the field distribution inside the $N$ laminae, as $\rho_{S}$ approaches zero and the ratio $\rho_{N} D / d=D_{n} / d$ of domain width to sample thickness increases. In fact, because of the flaring out of the field lines in the $N$ lamina at the sample surface, the field is certainly inhomogeneous within a depth of $\approx D_{n}$. One has $B=H_{c}$ near the domain wall, but $B$ is necessarily smaller in the "middle" region especially near the surface, ${ }^{1,7}$ the scale 


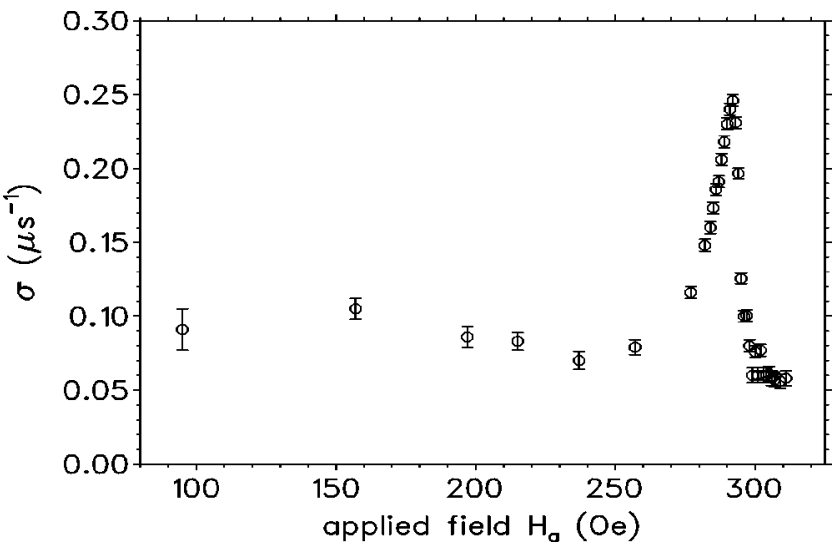

FIG. 4. Damping rate $\sigma$ vs $H_{a}$, upward field scan. The sharp asymmetric peak shows the field inhomogeneity just below the structural phase transition at $H_{t} \approx 295$ Oe (see Fig. 1). The damping rate in the $I$ state remains higher than $\sigma_{0} \approx 0.06 \mu \mathrm{s}^{-1}$ for the uniform $N$ state, due to the contribution of the $N-S$ interfaces.

of the variation of $B$ in the plane ortogonal to the laminae being $\approx D_{n}$. For example, at $H_{a} \approx 0.9 H_{c}$ the calculation ${ }^{7}$ predicts $\left(H_{c}-B\right) \approx 0.06 H_{c}$ at the middle line between the two interfaces. However, the observable width $\delta B$ $\approx\left[\overline{(B-\bar{B})^{2}}\right]^{1 / 2} \propto \sigma$ of the $\mu$ SR line remains negligible as long as the volume fraction $\propto D_{n} / d \approx(\delta / d)^{1 / 2} / \rho_{S}$ of the surface regions is small, and becomes large only with increasing $D_{n}$ as $\rho_{S} \rightarrow 0$. The increase of $\delta B$ with $H_{a}$ is abruptly stopped when the $S$ laminae disintegrate into threads near $H_{a}=H_{t}$. The value of $\sigma$ drops, since for the cylindrical geometry the field $B \approx H_{c}$ at the interfaces decays more rapidly to $\approx H_{a}$ in the inside of the $N$ regions, so that the volumes associated with $B \approx H_{c}$ are strongly reduced and their contribution to $\delta B$ becomes very small. (In downward field runs the $I$ state appears only for $H_{a}<287 \mathrm{Oe}$, the peak in $\sigma$ is shifted accordingly to the left.) A more quantitative treatment of the volume average $\delta B=\left[\overline{(B-\bar{B})^{2}}\right]$ as a function of $H_{a}$ would depend on the particular model ${ }^{1,7,10}$ for calculating the spatial distribution $\mathbf{B}(\mathbf{r})$ with curved boundaries at the surface region, it lies beyond the scope of this work.

Clearly, the field inhomogeneity in the $N$ domains for $260<H_{a}<295$ Oe could already be inferred from the dip in $\bar{B}$ seen in Fig. 3: the significant depression $\bar{B}<H_{c}$ of the average field is evidence of relatively large $N$ regions with $B$ values significantly below $H_{c}$, consistently with the observed behavior of $\sigma$. Further, it was found that the increased damping due to this spatial field structure is not exactly Gaussian (as indicated already by the anomaly in Fig. 2) and even less exponential. Since within the given time window of $\approx 12 \mu$ s the results obtained by exponential or Gaussian decay functions are very nearly the same and the fits equally good so long as the damping is weak $\left(\sigma<0.1 \mu \mathrm{s}^{-1}\right)$, and at the peak of the damping rate near and below $H_{a}=H_{t}$ (where the difference becomes appreciable) the Gaussian approximation was found to be slightly better, this was used overall for the analysis.

For $H_{a} \lesssim 260$ Oe the damping rate remains low and varies only weakly, $\sigma \approx \sigma_{I}=0.09 \mu \mathrm{s}^{-1}$ which is definitely larger than $\sigma_{0}$. The difference $\sigma_{i f}=\sigma_{I}-\sigma_{0}$ is due to the interfaces in the $I$ state. Here, on the $S$ side of the interface within a thickness of $\delta=\xi-\lambda_{L} \approx 10^{-5}-10^{-4} \mathrm{~cm}$, the field $B$ grows continuously from zero to $H_{c}$ leading to an additional "interface" broadening, absent in the uniform $N$ state. Since the volume ratio of the interface regions to the bulk of the $N$ domains is $V_{i f} / V_{N} \approx \rho_{S}(\delta / d)^{1 / 2}$, the "interface" contribution $\sigma_{i f}$ varies with $\rho_{S}$. Due to the smallness of $\sigma_{i f}$, its variation can become visible only far from the main peak at $H=H_{t}$. An increase of $\sigma$ with $\rho_{S}$ for $H_{a}<250$ Oe is indeed indicated in Fig. 4, but for a quantitative statement more accurate measurements in the low field region $\rho_{S}>0.5$ are needed.

It is to be pointed out that the above results were obtained for a high purity single crystal. In contrast, for the polycrystalline sample the abrupt jump of $B$ at $H_{a}=287$ Oe due to field supercooling is replaced by a smooth transition occurring much nearer to $H_{c I}$, at $H_{a}=295$ Oe. Also, only a vague indication for a change of the laminar structure near $H_{c I}$ can be seen and, opposite to the single crystal, the polycrystal shows a normal hysteretic behavior: the $B\left(H_{a}\right)$ path in the $I$ state near the $I \rightarrow N$ transition is not unique, different paths can intersect each other depending on the history.

\section{CONCLUSIONS}

The $\mu$ SR data on the internal magnetic field in single crystal white tin reveal several new features of the intermediate state near the $I \leftrightarrow N$ transition. The induction $\bar{B}$ in the $N$ domains just below the transition is by about 3\% lower than $H_{c}$, and the locally measured value $H_{c}=305.5 \pm 0.5$ Oe of the critical field is attained only by decreasing $H_{a}$ down to $H_{a} / H_{c} \lesssim 0.85$. On increasing $H_{a}$ in the intermediate state, the data indicate a reversible transition from the laminar to a threadlike phase at $H_{t}=295 \mathrm{Oe}$, before the $I \rightarrow N$ transition at $H_{c I} \cong 300 \mathrm{Oe}$. The damping rate $\sigma$, characterizing the width of the field distribution, rises sharply by a factor of $\approx 4$ as $H_{a}$ decreases below $H_{t}$, and forms a $\lambda$-like peak with a shoulder extending down to $H_{a} \approx 260 \mathrm{Oe}$. This is the same range where the difference $H_{c}-\bar{B}$ becomes important with the increase of $H_{a}$, showing the larger weight of regions with $B<H_{c}$ in the $N$ domains as the domain width increases. On decreasing $H_{a}$ below $\approx 260$ Oe, $\sigma$ remains significantly higher than $\sigma_{0}$ observed in the homogeneous $N$ state, reflecting the continuous field distribution within the $S-N$ domain interfaces. In downward field runs, the uniform $N$ state persists supercooled down to $H_{a}=H_{c I^{\prime}}=287$ Oe, which corresponds to $\left(H_{c}-H_{c I^{\prime}}\right) / H_{c} \approx 6 \%$.

\section{ACKNOWLEDGMENTS}

We are indebted to V.F. Gantmakher for providing us with the tin crystal, to A. Schenck for a valuable discussion, and to the PSI staff members for the excellent muon beam. One of the authors (V.E.) is grateful for Grant No. 98-0217142 of the Russian Fund for Fundamental Research. 
${ }^{1}$ L.D. Landau and E.M. Lifshitz, Electrodynamics of Continuous Media (Pergamon, Oxford, 1984).

${ }^{2}$ E.R. Andrew, Proc. R. Soc. London, Ser. A 194, 98 (1948).

${ }^{3}$ M. Tinkham, Introduction to Supercoductivity (McGraw, New York, 1996).

${ }^{4}$ P.G. de Gennes, Superconductivity of Metals and Alloys (Benjamin, New York, 1966).

${ }^{5}$ J.D. Livingstone and W. DeSorbo, in Superconductivity, edited by R.D. Parks, (Dekker, New York, 1969), Vol. 2.

${ }^{6}$ M. Desirant and D. Shoenberg, Proc. R. Soc. London, Ser. A 194, 63 (1948).

${ }^{7}$ A. Fortini and E. Paumier, Phys. Rev. B 5, 1850 (1972).

${ }^{8}$ R.P. Huebener, Magnetic Flux Structures in Superconductors (Springer, Berlin, 1979).

${ }^{9}$ A.T. Dorsey and R.E. Goldstein, Phys. Rev. B 57, 3058 (1998).

${ }^{10}$ C.R. Reisin and S.G. Lipson, Phys. Rev. B 61, 4251 (2000).

${ }^{11}$ L.D. Landau, J. Phys. (USSR) 7, 99 (1943).

${ }^{12}$ P.R. Solomon and R.E. Harris, in Proceedings of the 12th International Conference on Low Temperature Physics, edited by E. Kanda (Academic Press of Japan, Kyoto, Japan, 1971), p. 475.

${ }^{13}$ M. Gladisch, D. Herlach, H. Metz, H. Orth, G. zu Putlitz, A.
Seeger, H. Teichler, W. Wahl, and W. Wigand, Hyperfine Interact. 6, 109 (1979).

${ }^{14}$ V.G. Grebinnik, I.I. Gurevich, V.A. Zhukov, A.I. Klimov, L.A. Levina, V.N. Maiorov, A.P. Manych, E.V. Mel'nikov, B.A. Nikol'skii, A.V. Pirogov, A.N. Ponomarev, V.S. Roganov, V.I. Selivanov, and V.A. Suetin, Sov. Phys. JETP 52, 261 (1980).

${ }^{15}$ V.S. Egorov, G. Solt, C. Baines, D. Herlach, and U. Zimmermann, Physica B 289-290, 393 (2000).

${ }^{16}$ V.F. Gantmakher (private communication).

${ }^{17}$ A. Schenck, Muon Spin Rotation Spectroscopy (Hilger, Bristol, 1986).

${ }^{18}$ R. Abela, A. Amato, C. Baines, X. Donath, R. Erne, D.C. George, D. Herlach, G. Irminger, I.D. Reid, D. Renker, G. Solt, D. Suhi, M. Werner, and U. Zimmermann, Hyperfine Interact. 120-121, 575 (1999).

${ }^{19}$ J.A. Osborn, Phys. Rev. 67, 351 (1945).

${ }^{20}$ Handbook of Chemistry and Physics, 79th ed. (CRC Press, Boca Raton, 1998).

${ }^{21}$ Handbook of Physical Quantities, edited by I.S. Grigoriev and E.Z. Meilikhov (CRC Press, Boca Raton, 1997). 